\title{
Research on P2P flow identification model based on communicating network topology
}

\author{
Sun Zhixin ${ }^{1 \star}$, Yan Xiaojuan ${ }^{2}$ and Chen Songle ${ }^{1}$ \\ ${ }^{1}$ College of Computer of Nanjing University of Posts and Telecommunications, Nanjing 210003, China. \\ ${ }^{2}$ Key Laboratory of Broadband Wireless Communication and Sensor Network Technology, Ministry of Education, \\ Nanjing University of Posts and Telecommunications, Nanjing 210003, China.
}

Accepted 27 June, 2011

\begin{abstract}
This paper presents a new P2P (Peer-to-Peer)-CNTIM model for identifying P2P traffic based on the special characteristics of P2P communication network topology. P2P-CNTIM model identifies P2P flow by combining the characteristic of multi-hosts with the characteristic of the type of the other communicating peer so as to improve the identification accuracy and efficiency. It provides the critical detecting technique of the type of other communicating party and sets up assessment functions, scheduling mechanism and the core process of operating this P2P-CNTIM model. The experimental results showed that P2P-CNTIM model system has a lower misjudgment rate and higher identification accuracy. Furthermore, the implementation efficiency of the prototype system is obviously improved in comparison with the P2P identification method of the traditional payload characteristics.
\end{abstract}

Key words: P2P, flow identification, communicating network topology.

\section{INTRODUCTION}

P2P (Peer-to-Peer), risen on the 20th century, is a new distributed communication and application model which is different from the traditional $\mathrm{C} / \mathrm{S}$ (client/server) model. P2P-flow analysis and identification technology can basically be divided into six categories: Port identification technology; payload-based feature recognition; pseudoparticipation technology of $\mathrm{P} 2 \mathrm{P}$ traffic identification; flow feature recognition; cross-layer recognition and machine learning-based P2P streaming identification technology.

There are some forerunners on this topic. Subhabrata and Jia (2004) proposed port flow identification technology. But recently, many $\mathrm{P} 2 \mathrm{P}$ protocols with dynamic port technology could avoid detection; this technology is not practical at this stage. The stream detection method, payload-based technology in Xing et

${ }^{*}$ Corresponding author. E-mail: sunzx@njupt.edu.cn

Abbreviations: P2P, Peer-to-peer; C/S, client/server; NAT, network address translation; FTP, file transfer protocol; TCP, transmission control protocol; UDP, user datagram protocol; ICMP, internet control message protocol; IP, internet protocol. al. (2007), Feng et al. (2009), jing et al. (2007), Rui (2007) and Holger et al. (2006a,b) has advantage of directly perceived, high efficiency and low false detection rate. Xing et al. (2007) discussed the detective method base-on content redistributed features which only can detect known P2P protocol and P2P stream but fail to test the unknown P2P flow; Feng et al. (2009), jing et al. (2007) and Rui (2007) carried out the P2P flow detection, respectively through collecting statistical package length and the basic characteristic quantities and gathering the behavior characteristics. Holger et al. (2006a,b) conduct an investigation into P2P analysis and detection in multiprotocol environment. The chief objection to these methods payload-based is that, it may involve confidentiality or privacy issues; for this reason, there is social controversy. Cross-layer identification techniques in Chunzhi et al. (2009) are widely used in business, which combines payload feature and flow characteristics detection to avoid narrow conclusion. However, largescale flow detection is lack of practicability and it needs further research. In view of complexity and dynamic nature of network traffic itself, the machine learning research for classifying network traffic application is still in its infancy. 


\section{CHARACTERISTICS OF COMMUNICATION NETWORK TOPOLOGY}

P2P-CNTIM for identifying P2P traffic based on the special characteristics of $\mathrm{P} 2 \mathrm{P}$ communication network topology. P2P-CNTIM model identifies P2P flows by combining the multi-hosts characteristic with the characteristic of the type of the other communicating party of P2P so as to improve the identification accuracy and efficiency. As accuracy identification of P2P stream or non-P2P stream is the foundation to further research, we need to find out some characteristics which are the key factors of identification while analyzing the characteristics of P2P network topology. In other words, we need to locate some deterministic characteristic that can directly distinguish the P2P stream or non-P2P stream. These characteristics are generally shared by ordinary P2P applications.

The network topology structures of P2P application are: Multi-hosts characteristic. By "multi-hosts characteristic" we mean a feature that P2P nodes communicate with multiple hosts for a period of time or at a certain moment. P2P applications have generally adopted a different port while communicating with different target host (Thomas et al., 2004), therefore, we can choose a threshold that when the number of nodes which an observed node's peer communicated with and different communication ports is greater than within a certain time, it can be said that the observed host has generated P2P flow.

The type of the other communicating party of P2P characteristic [the communication features of inner net within network address translation (NAT) traversal]. The other communicating party of P2P application includes within the network after NAT hosts also contains the hosts with an independent public network internet protocol (IP). This paper calls this feature as the type of the other communicating party characteristic. When the number of the inner net host after NAT communicated with observed node is greater than a certain threshold, it may be considered that the host had a P2P streaming.

Characteristic of communicating with public network IP host. P2P application, in the process of connecting with the public network, needs public network server as medium of NAT traversal; in other words, part of the nodes communicated with observed node are the nodes with public network IP.

Besides these network topology features, P2P streaming also has significant statistical features, such as interactive data features, data packet length characteristics, typical P2P application ports characteristics, communication protocols characteristics, average flow rate characteristics, flow time characteristics and the SYN/ACK statistical features of P2Ptransmission control protocol (TCP).

By analyzing the various characteristics, the paper chooses multi-hosts characteristic and the type of the other communicating party of $\mathrm{P} 2 \mathrm{P}$ characteristic as the basis for identification of P2P streaming. The first feature is relatively easy to handle, but the follow characteristic needs adopting a practical technology to be identified. That is to say, it needs some technology to distinguish that the other communicating party is inner net host after NAT device or the host is providing internet services with independent public network IP.

\section{P2P-CNTIM MODEL INTRODUCTION}

P2P-CNTIM model, shown in Figure 1, includes traffic anomalies detection module, data packet queues store module, multi-host feature statistics module, type of the other communicating party characteristic statistics module and flow control module. Traffic anomalies detection module collects data packet and puts the data packets which fit certain requirements into the queue and data packet queues store module will store the qualified data packet. If the bandwidth of detected host exceeds the limit threshold of user bandwidth, the data packet in queue will be judged by P2P-CNTIM model's discriminant function of statistical characteristic. Either of the multihosts statistical values collected by multi-hosts feature statistics module or the other statistical values gathered by the type of the other communicating party of P2P characteristic statistics module meets demand, then mark the packet as P2P flow and put the P2P flow into flow control module and do some processing. On the other side, if neither of the two characteristic statistics is qualified, and then mark the data packet as non-P2P flow and take some normal process. The first part of this chapter will introduce a key technology of acquiring the type of other communicating party. The follow part will detailed discussion on the P2P-CNTIM model's discriminant function of statistical characteristic. The last two parts will describe the scheduling mechanism including excitation condition of detection process and so on.

\section{Key technology of acquiring the type of the other communicating party}

P2P-CNTIM model identifies P2P flows by combining the multi-hosts characteristic with the characteristic of the type of the other communicating party of P2P so as to improve the identification accuracy and efficiency. The first feature is relatively easy to handle, but follow characteristic needs of some technology to distinguish that, the other communicating party is inner net host after NAT device or the host providing internet services with independent public network IP. P2P flow identification system usually located in the near gateway device (we suppose that the software works on the gateway), the observed node belongs to the host of inner net, and the host communicated with inner net host may be the host with independent public network IP or an inner host after NAT device, as shown in Figures 2 and 3. 


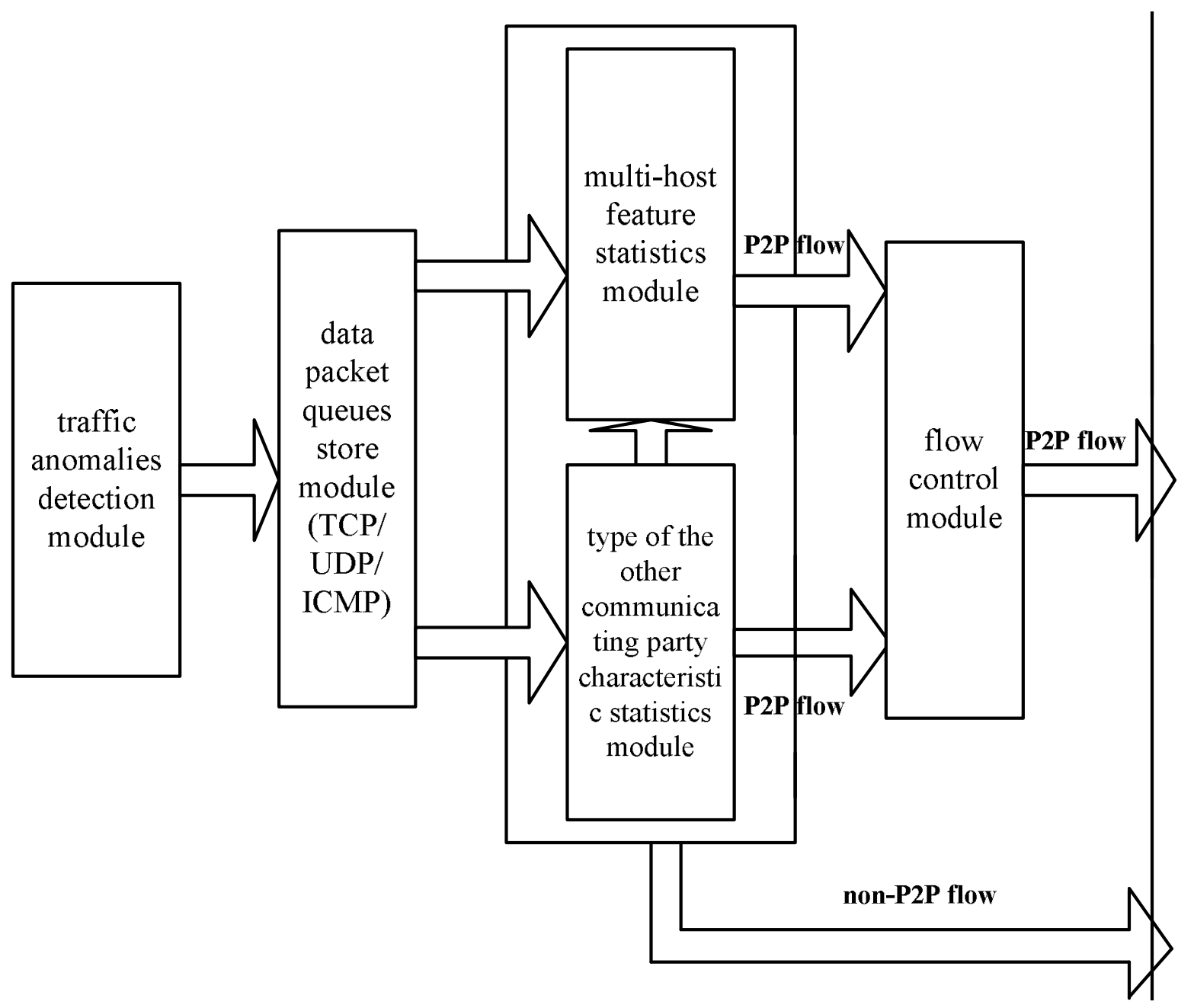

Figure 1. P2P-CNTIM model.

As seen in Figures 2 and 3 , the data packets $A$ and $B$ will show the same destination address, final address and source address on the gateway. But actually, this is not in accordance with the fact. Specifically, the data packet $A$ was generated by the host with public network IP of 202.138.24.35, while data packet $B$ was generated by the inner net host of 192.168.1.100. In order to acquire the actual address of the data packet, we import the "TTL" technology [Peter Phaal. Detecting NAT Devices using sFlow (EB/OL)] and network path length to solve the problem. In Figure 2, we can see that the host with IP of 202.138.24.35 sent packet $A$ to the internet, every time the packet jump through the internet, the TTL value will decrease by 1 . So when packet $A$ arrive its destination address of gateway $A$, the reduction of TTL values actually represent the number of hops passing the path (Here, the number of hops can be used to represent the network distance), TTL decrease values and actually network distance of from host to gateway $A$ is the same. Similarly, as shown in Figure 4, the reduction in TTL value of packet $B^{\prime}$ is $64-53=11$, while in ping operation, the TTL of response was reduced to $64-54=10$, the two TTL decrease value are not equal, so we are sure the 202.102.24.35 is not the real host that produced the data packet $B$.

According to the calculation principle of TTL; if TTL reduction value of received packet is greater than the network distance of receiving end to network device (host) corresponding to IP address of this packet, it can consider that the source IP address shown by the packet are not the real IP address, that is to say, type of the other communicating party of the packets' real host is after the NAT device.

With the technology to get the packet generated by gateway $B$, it seems to know the hops of gateway $A$ and $B$, thus; we can use the below measures:

Gateway A sent ping packet to gateway $B$, according to the TTL value of response result of ping operation to calculate the number of hops.

Gateway $A$ sends user datagram protocol (UDP) packet of a port to gateway $B$, then gateway $B$ will return internet control message protocol (ICMP) packet of a not exist 


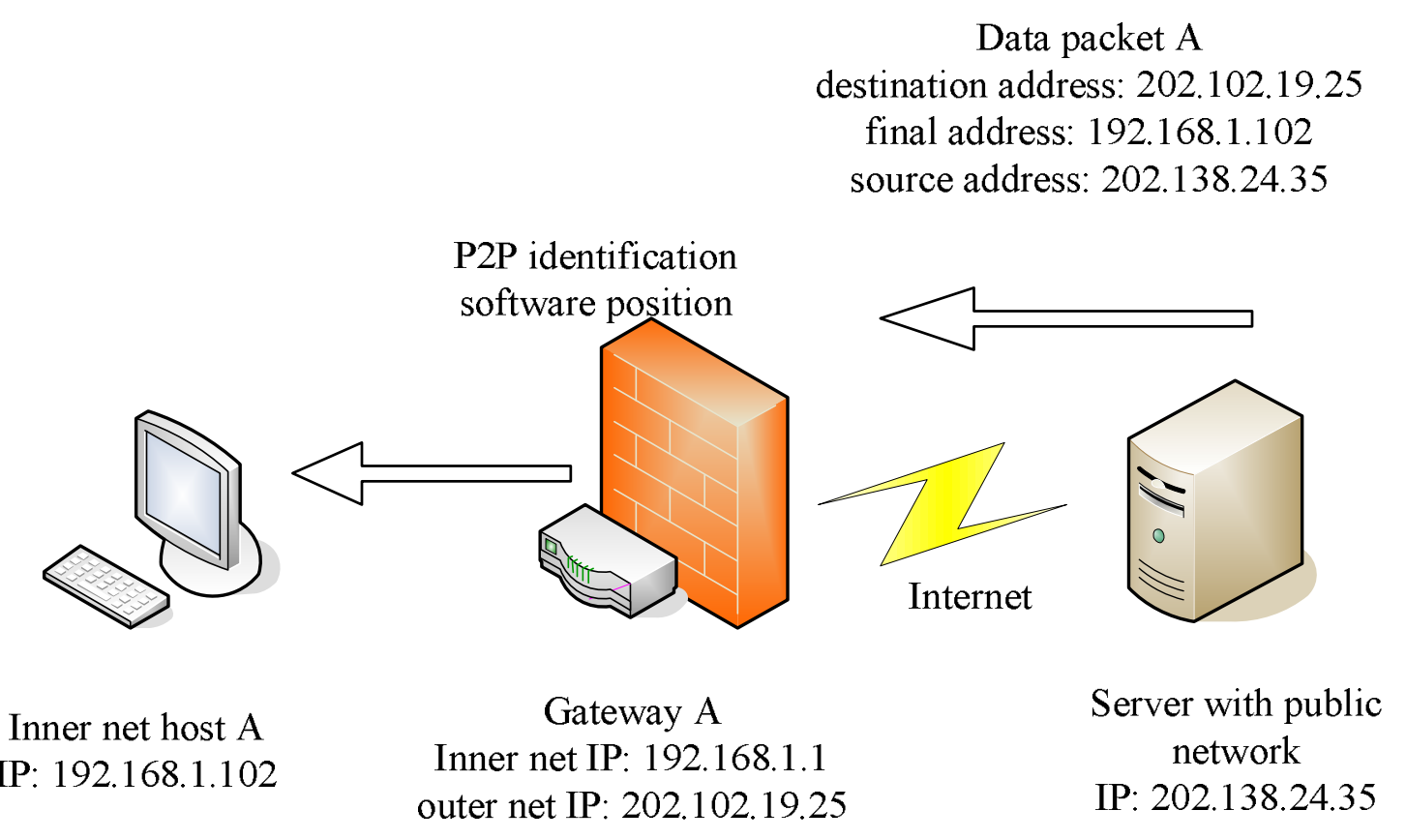

Figure 2. Inner net host communicate with the host with independent public network IP.

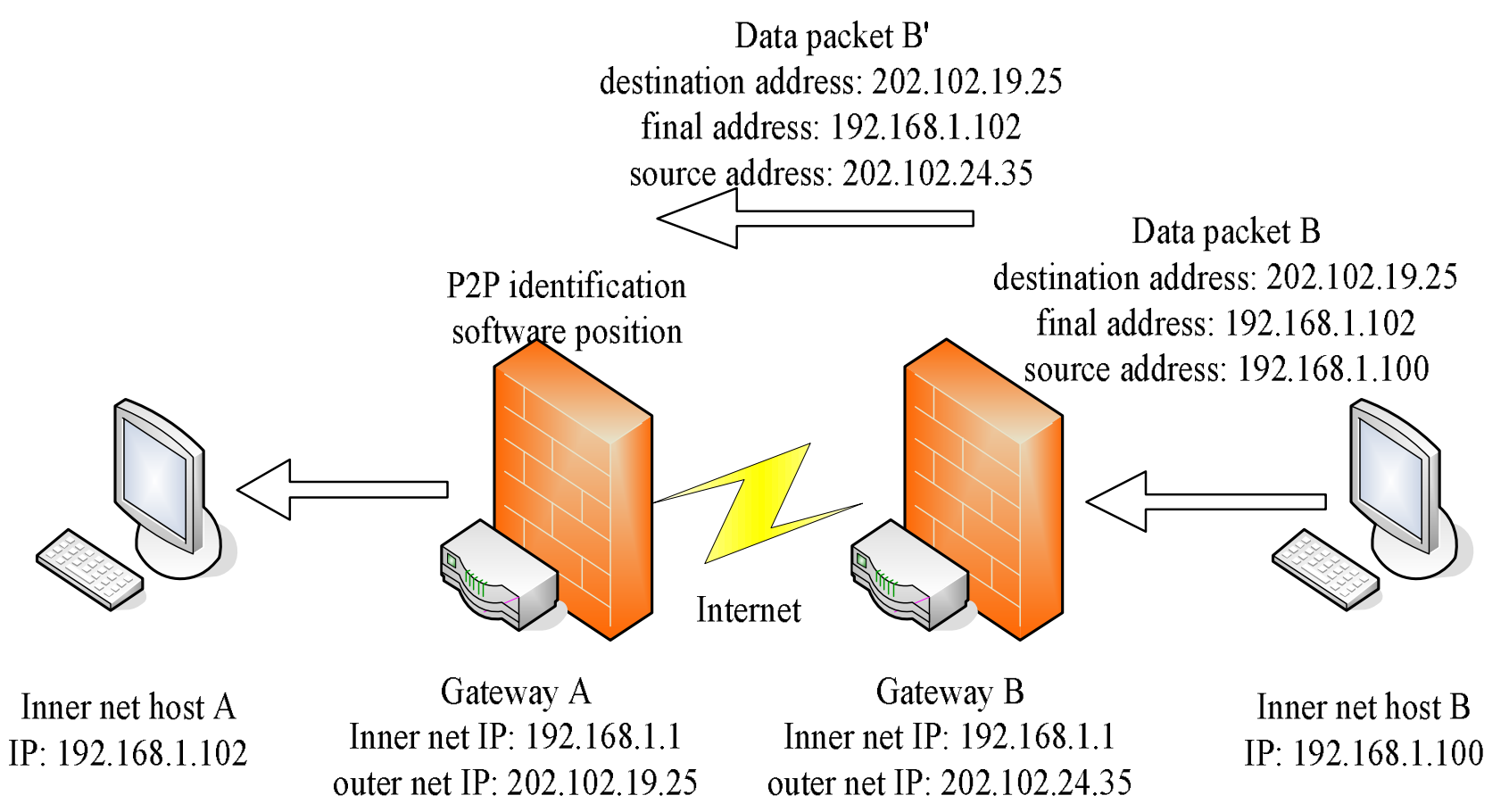

Figure 3. Inner net host communicate with the inner net host after NAT device.

port, and according to the TTL value of returned results to calculate the number of hops.

Gateway A sends TCP connection packet of a large port to gateway $B$, then gateway $B$ will return RST packet of TCP, and according to the TTL value of returned results to calculate the number of hops;
As to reduce false-positive rate, we add the following two constraints on the algorithms of calculating the distance between the two gateways:

Constraint 1: Only detect the host with communication port greater than 10000 , because mapping port of NAT 

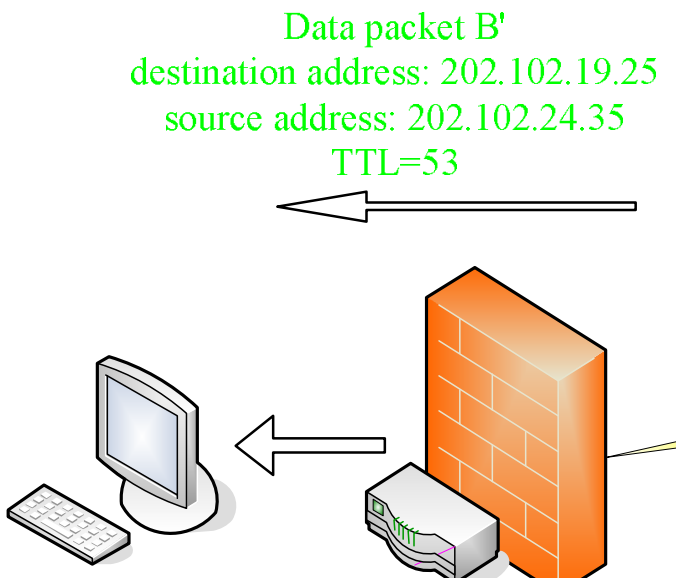

-

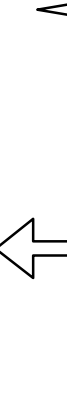

?

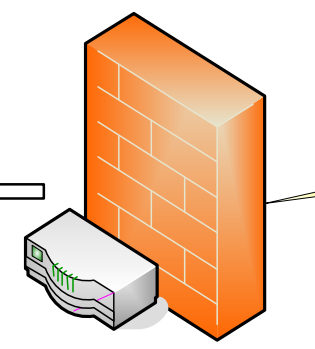

Inner net host $\mathrm{A}$

IP: 192.168.1.102

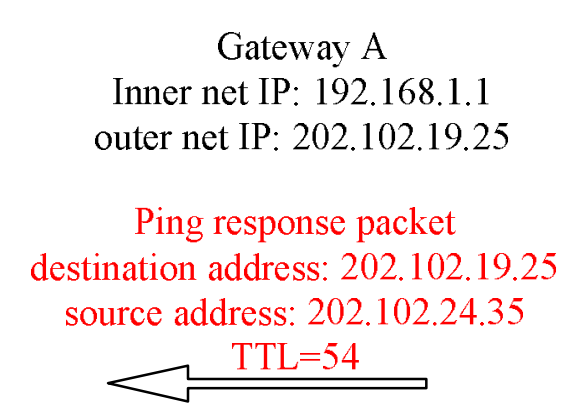

Internet

10 jump

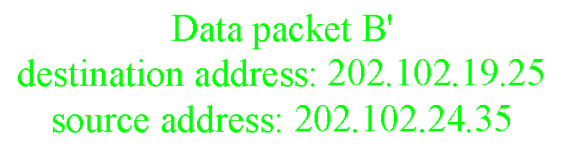

source address: 202.102.24.35

$\mathrm{TTL}=63$

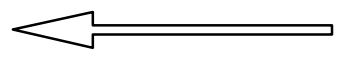

destina

destination address: 202.102.19.25

final address: 192.168.1.102

source address: 192.168 .1 .100

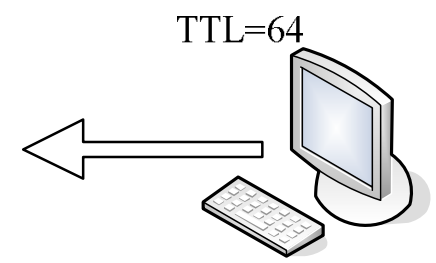

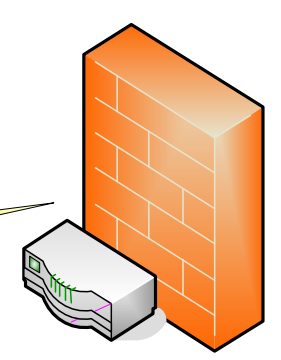

Gateway B

Inner net host B

Inner net IP: 192.168.1.1 outer net IP: 202.102.24.35

IP: 192.168.1.100

Ping response packet

destination address: 202.102.19.25

source address: 202.102.24.35

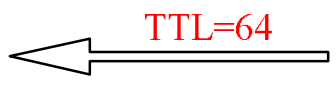

Figure 4. The different TTL decrease values of inner net host.

device is generally high-port. Adding this constraint is not only to reduce the system cost, but also avoid misjudgment of common network host providing WEB and file transfer protocol (FTP).

Constraint 2: Using the above three rules for testing at the same time, only test results are the same that can sure the type of the other communicating party being after the NAT device. Also, this constraint can reduce false-positive rate.

These additional constraints actually make the process of acquiring type of the other communicating party to be low false-positive rate and miss ratio while P2P-CNTIM model adopt very small threshold. In other words, as long as it is ensured that a few part of type of the other communicating party is after NAT device, according to the rigor of algorithm, we are sure the data flow is P2P stream. Even in the worst case, using the characteristic of the type of the other communicating party cannot identify as the P2P flow, since P2P-CNTIM model makes use of the combination of the multi-hosts characteristic with the characteristic type of the other communicating party of P2P so as to improve the identification accuracy and efficiency, we also can utilize multi-hosts characteristic to classify the P2P flow correctly.

After we get the key technology of acquiring the type of the other communicating party, we can easily gain the multi-hosts statistical values and the statistical values of the type of the other communicating party, and then we need establishing some discriminant function to judge the value for P2P identification.

\section{P2P-CNTIM model's discriminant function of statistical characteristic}

The definition of discriminant function of multi-hosts statistical characteristic P2P applications generally communicate with a large number of nodes, so that the number of hosts communicated usually is greater than a threshold, but the results of different source port and the port of the other communicating party are close to the IP of the other communicating party. In accordance with P2P multi-hosts characteristic, in order to reduce falsepositives rate, therefore, we add some port restrictions to multi-hosts connectivity features.

Definition 1: Multi-hosts statistical characteristic variables dest_ip: the address of the other communicating party connected with observed host

diff_dest_ip_count: the number of different IP address of the other communicating party in a period of statistics time

dest_port: a port of the other communicating party connected with observed host: 
diff dest port count: the number of different port of the other communicating party in a period of statistics time src_port: the source port to connect with observed host diff_src_port_count: the total number of local source port of observed host

\author{
Definition 2: Discriminant function of multi-hosts \\ characteristic \\ diff_dest_ip_count $>n 1$ \\ diff_dest_port_count $>\mathrm{n} 2$ \\ diff_src_port_count $>$ n3 \\ diff_dest_port_count diff_dest_ip_count

In formula, n1, n2, n3 are discriminant threshold, the last condition represent the number of different port of the other communicating party and the number of different IP address should be similar, as even the observed P2P application communicate with many hosts through NAT traversal, but every node of the other communicating party will be mapped to public network IP and port by NAT device. Obviously, these ports are different, and nodes of the other communicating party are unlikely to belong to the same NAT, so for the current observed node, (1) hold, in other words, as long as the above conditions are satisfied, we are sure that the flow is P2P stream.

The definition of discriminant function of type of the other communicating party statistical characteristic

Definition 3: Statistical characteristic of type of the other communicating party variables

desp_ip_nat: The number of inner net after NAT device connects to observed host.

dest_ip_internet: The host of the other communicating party is host with public network IP.

dest_ip_nat_count: The host of the other communicating party is host within NAT traversal.

After receiving the packet from the other communicating party, use the key technology to ascertain the type of the other communicating party, only if the number of the hosts of the other communicating party within NAT traversal is larger than $n 4$, then the data traffic can be considered as P2P flow.

Definition 4: Discriminant function of the type of the other communicating party characteristic:

dest_ip_nat_count>n4.

\section{P2P-CNTIM model's discriminant function of statistical characteristic}

For P2P-CNTIM model, the relationship of multi-hosts characteristic and the type of the other communicating party characteristic is "or" relationship, that is to say, either of the two characteristic statistics meets demand, then mark the data flow as P2P flow.

Definition 5: P2P-CNTIM model's discriminant function of statistical characteristic

dest_ip_nat_count $>n 4 \|\{($ diff_dest_ip_count $>n 1) \&$ (diff_dest_port_count $>$ n2)\&(diff_src_port_count $>$ n3)\&

(diff_dest_port_count diff_dest_ip_count)\} Scheduling mechanism of P2P-CNTIM model

For data packet collected during a period of time, model can judge the data whether Equation 3 is satisfied to identify the P2P flow, the formula determines statistics variables and the objective of statistical process, it is also the core discriminant function of P2P-CNTIM model. Of course, in the whole identification process, there is also need to establish the scheduling mechanism of the model.

Definition 6: Correlation variable of scheduling mechanism of P2P-CNTIM model

packet_valid_time: The valid time of the data packet recv_packet_time: Packet received time

limit_bandwidth: User network bandwidth limit control time: P2P flow control time of destination host P2P interval_time: Time interval of destination host which is identified as P2P flow

constant_value: Adjust the constant in flow control time.

System defined a current time, when recent time mirus recv_packet_time is larger than packer_valid_time, the data is invalid, if put the packet from some inner net host into the queue in the light of time order, then packet_valid_time determine the length of this queue, but if packet_valid_time is too long, the queue will be too large, it will lead to delay of the statistical results. On the other hand, if packet_valid_time is too short, the number of packet collected will be too little, and then statistical sample will be too little, it also affects the statistics result, therefore, it is necessary to choose an appropriate packet_valid_time. In the network plan, it needs to check user average bandwidth usage, just as limit_bandwidth, we can use this value as P2P-CNTIM scheduling driver, if user current bandwidth is greater than the user limit bandwidth, then start P2P flow identification model, or else, do not start it. When inner host was identified as P2P application, then the model needs to control the network behavior on that host. After having identified the P2P flow, in a certain time range, the target host will be protocol blocked by P2P identification system so that the system can limit the bandwidth of the host. For every host of inner net IP, the control_time is a dynamically change process, it is adjusted based on the user's network condition.

Definition 7: P2P-CNTIM flow control time adjustments

temp_time = P2P_interval_time/constant_time

control_time $=$ control_time/temp_time

control_time $=\max$ (default_control_time,control_time)

In Equation 4, it can be known that the P2P_interval_time 
and constant value are the multiple relationships, which is temp_times. If temp_times $>1$, then control_time will become less, that is to say, the P2P interval time is larger, and then the control_time will be smaller. The last expression of Equation 4 cannot be smaller than default minimum value of system definition.

Through the above algorithm, we can dynamically restrict the $\mathrm{P} 2 \mathrm{P}$ flow according to usage condition, the oftener usage of P2P application, the more control time imposed on users.

\section{PERFORMANCE ANALYSIS}

We carry out the P2P experiments in a laboratory of a campus where we choose twenty of the hosts which formed a local area network, and then dispose this system on a server host; the whole system test environment is shown in Figure 6.

\section{False-positive rate analysis and test}

\section{A. The false-positive rate test based on multi-hosts characteristic}

To ensure recognition accuracy, the basic principle of P2P-CNTIM flow identification model is to keep the falsepositive rate as low as possible, and then detect the traffic according to Equation 1 based on multi-hosts characteristic. Before the experiment, set the packet_valid_time as a unit time. Through the discussion test prove, take $n 2=n 1{ }^{*} 50 \%, n 3=n 1{ }^{*} 30 \%$, this formula obviously does not result in misjudgment to traditional web applications. And then we can get that $\mathrm{n} 1$, which can be confirmed a proper value through the test of non-P2P stream such as HTTP and FTP or so on, is a judge threshold based on multi-hosts detection in a unit time.

During the test process, 8 hosts in laboratory generated WEB FTP and other traditional network traffic, we set user limit bandwidth of every host to $25 \mathrm{k}$, and set that when a data stream is identified as P2P streaming, its control time is a unit time, and when the control time ends the data stream will be marked as normal. So that when a data stream is identified as P2P streaming, because the data packet queue contains one unit time of packet, this part of data packets can be considered as P2P packet stream. And we set the control time as unit time, so in the following unit time, the data packets of the host are also considered as P2P packet stream. We take ration of P2P data packets within these two units and total packets generated by 8 hosts represented the false-positive rate. While $\mathrm{n} 1$ gets different values, the false-positive rate of traditional network application is shown in Figure 7.

As seen from Figure 7, as $\mathrm{n} 1$ increases, thus resulting in reduction of false-positives rate, and its effect of false- positive rate is also getting smaller and smaller, when $\mathrm{n} 1$ is 20 , the obtained false-positive rate is less than $0.7 \%$, so when $\mathrm{n} 1$ is taken as 20 , P2P stream detection based on multi-hosts characteristic can get a lower falsepositive rate.

\section{B. The false-positive rate test based on the type of the other communicating party characteristic}

To detect the false-positive rate based on the type of the other communicating party characteristic, we need to obtain a threshold, n4. But consider the stringency of the judge function (port number $>10000$, etc). It can obtain a lower false-positive rate when $\mathrm{n} 4$ only takes a very small value, shown in Figure 8.

According to Figure 8, as n4 increases, thus resulting in reduced of false-positives rate, and its effect of falsepositive rate is also getting smaller and smaller, when $\mathrm{n} 4$ is 1 , the obtained false-positive rate is less than $0.4 \%$, so when $\mathrm{n} 4$ is taken as 1, P2P stream detection based on the type of the other communicating party characteristic can get a lower false-positive rate.

As mentioned before, P2P identification detection system first needs to ensure low false-positive rate, on this basis it needs to further improve recognition accuracy, as well as identifications, the most P2P stream and take them under control, it can result in good return. According to analysis and test results in this section, for Equations 1 and 2, we can get the thresholds, respectively, $\mathrm{n} 2=\mathrm{n} 1{ }^{*} 50 \%, \mathrm{n} 3=\mathrm{n} 1{ }^{*} 30 \%, \mathrm{n} 1=20, \mathrm{n} 4$ $=1$, this set threshold can ensure the system's falsepositive rate less than $0.7 \%$ which is the required standard.

\section{Accuracy rate analysis and test}

Recognition accuracy mainly represents system identification capacity of P2P stream. We can get a profound knowledge of P2P-CNTIM model by comparing P2P-CNTIM prototype system accuracy rate with the identification system based on payload feature accuracy rate while by comparing the two different P2P flow detection methods of P2P-CNTIM prototype system.

\section{The core process of P2P-CNTIM model}

As shown in Figure 5, we can get the core process of P2P-CNTIM model. Traffic anomalies detection module is collecting data packet and putting the data packets which fit Definition 6 into the queue and data packet queues module stores the qualified data packet. If the bandwidth of detected host exceeds the limit threshold of user bandwidth, the data packet in queue will be judged by P2P-CNTIM model's discriminant function of statistical characteristic. Either of the multi-hosts statistical values 


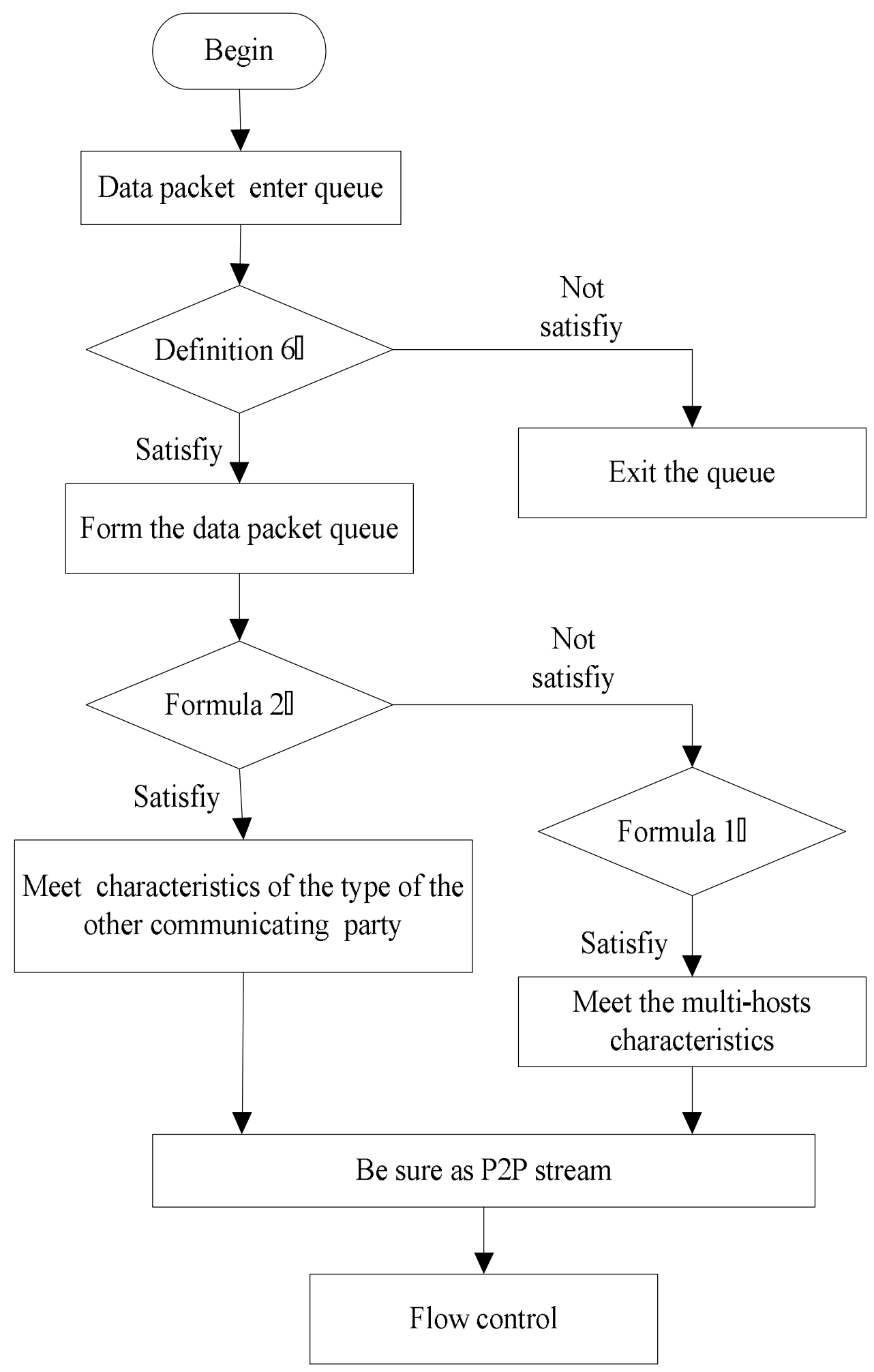

Figure 5. The core process of P2P-CNTIM model.

collected by multi-hosts feature statistics module or the other statistical values gathered by type of the other communicating party of P2P characteristic statistics module meets demand, then mark the packet as P2P flow and put the P2P flow into flow control module and do some processing. On the other side, if neither of the two 
Data packet $\mathrm{B}^{\prime}$ destination address: 202.102.19.25 source address: 202.102.24.35 TTL $=53$

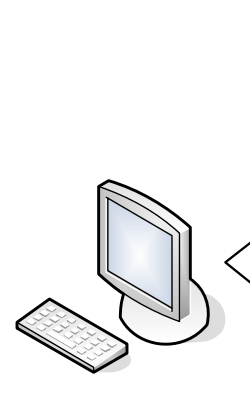

Inner net host $\mathrm{A}$ IP: 192.168.1.102

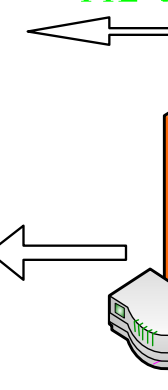

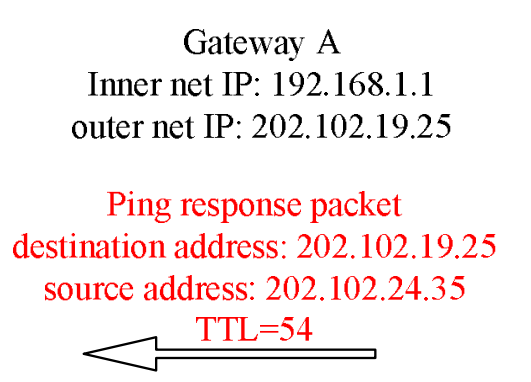
Data packet B'
destination address: 202.102 .19 .25
source address: 202.102 .24 .35

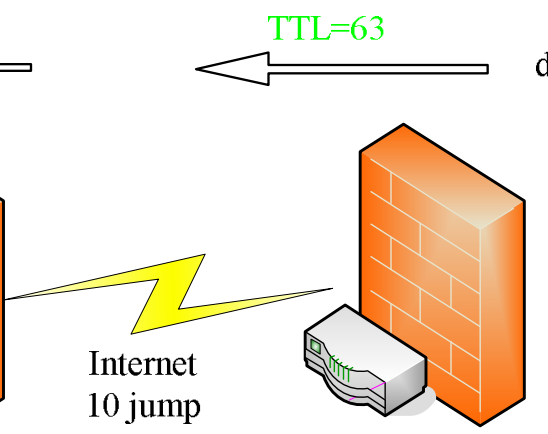

Data packet B
destination address: 202.102 .19 .2
final address: 192.168 .1 .102

source address: 192.168.1.100

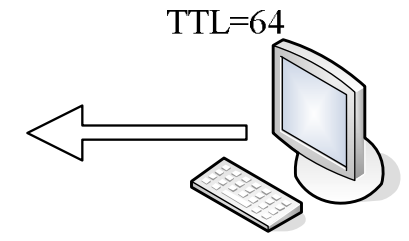

Gateway B

Inner net IP: 192.168.1.1 outer net IP: 202.102.24.35

Inner net host B
IP: 192.168.1.100

Ping response packet destination address: 202.102.19.25 source address: 202.102.24.35

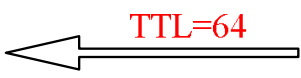

Figure 6. P2P-CNTIM prototype system test network structure.

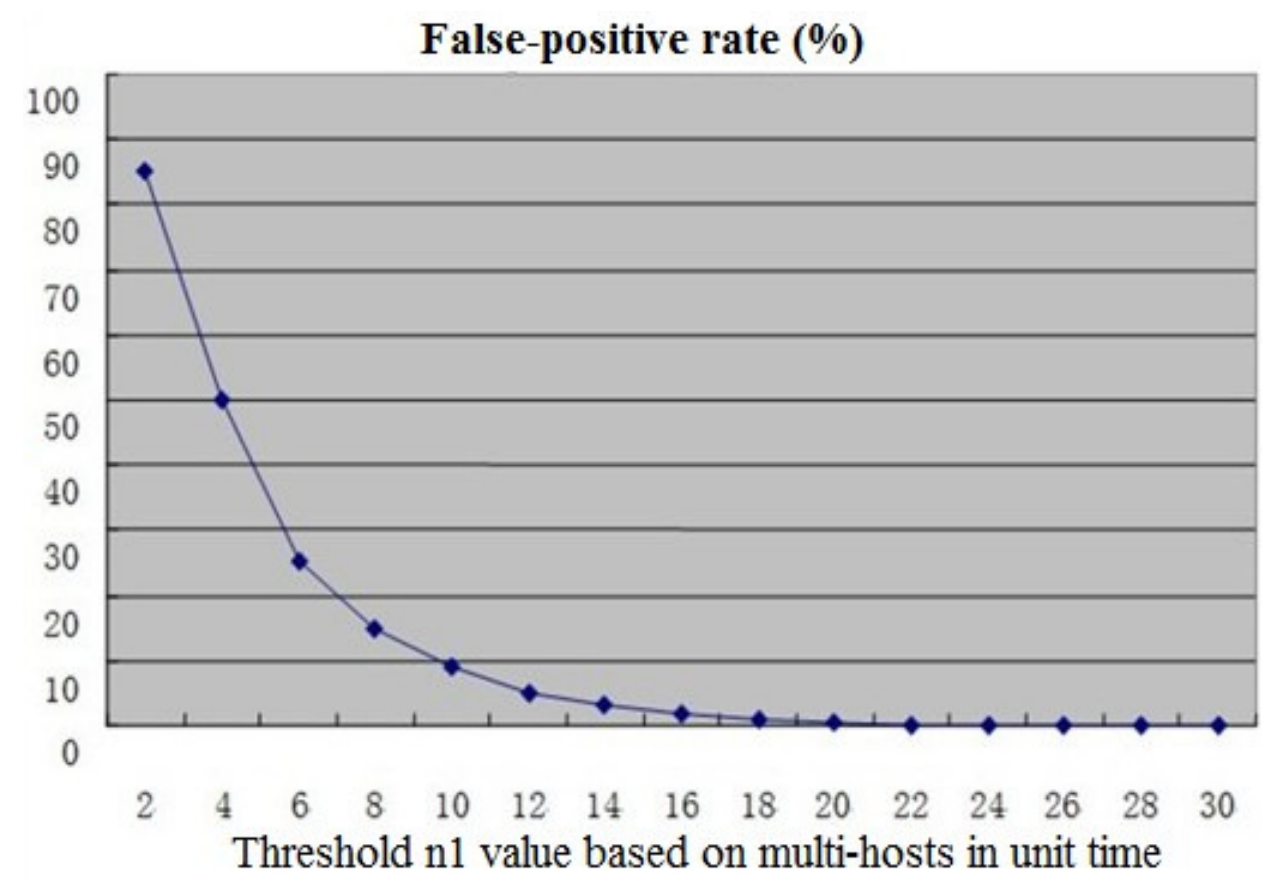

Figure 7. False-positive rate detection of P2P model based on multi-hosts characteristic.

characteristic statistics is unqualified, and then mark the data packet as non-P2P flow and take some normal process.
Here, we need do some explanation, the relationship of multi-hosts characteristic and the type of the other communicating party characteristic is "or" relationship, 


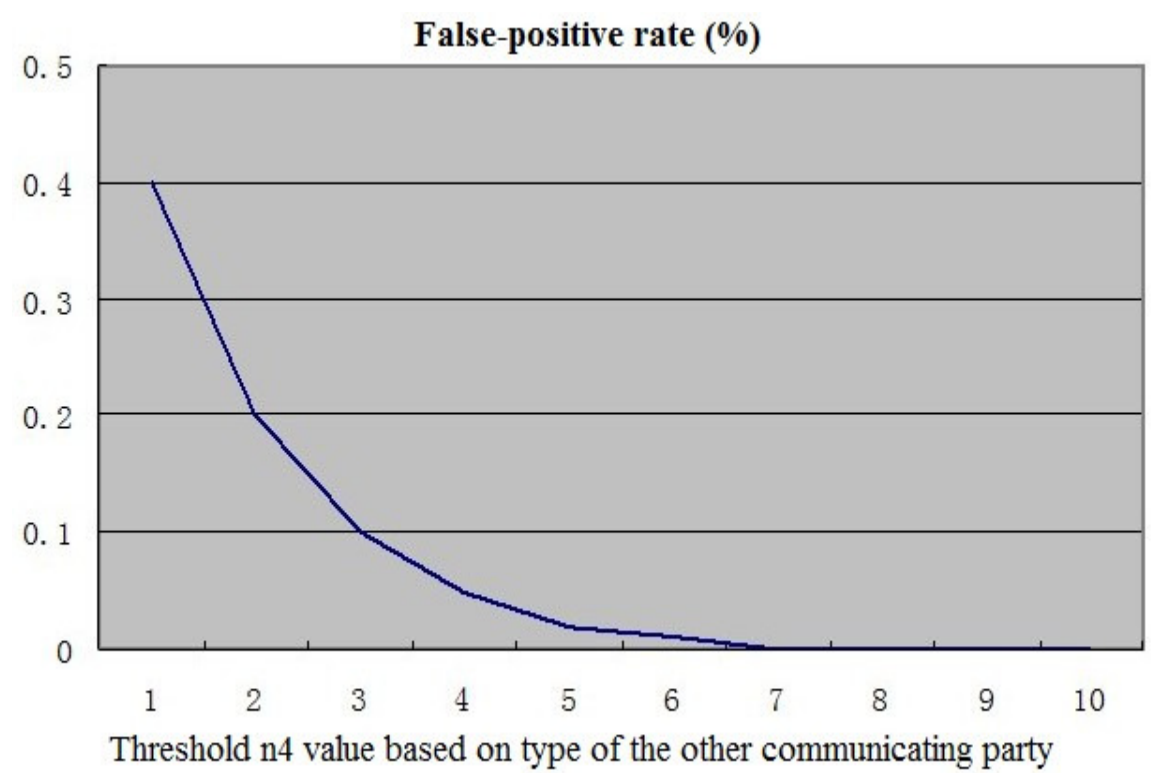

Figure 8. False-positive rate detection based on the type of the other communicating party characteristic.

Table 1. P2P payload-based system and P2P-CNTIM prototype system accuracy rate contrast.

\begin{tabular}{|c|c|c|c|c|c|}
\hline \multirow[b]{2}{*}{$\begin{array}{l}\text { Order } \\
\text { number }\end{array}$} & \multirow[b]{2}{*}{$\begin{array}{l}\text { Application } \\
\text { name }\end{array}$} & \multirow{2}{*}{$\begin{array}{l}\text { Existing payload-based } \\
\text { identification system } \\
\text { accuracy rate (\%) }\end{array}$} & \multicolumn{3}{|c|}{ P2P-CNTIM system P2P identification accuracy rate } \\
\hline & & & $\begin{array}{l}\text { Type of the other party } \\
\text { characteristic (\%) }\end{array}$ & $\begin{array}{c}\text { Multi-hosts } \\
\text { characteristic (\%) }\end{array}$ & Total (\%) \\
\hline 1 & BitTorrent & 91.25 & 46.53 & 37.26 & 83.79 \\
\hline 2 & eMule & 90.32 & 50.27 & 36.89 & 87.16 \\
\hline 3 & pplive & 88.94 & 42.35 & 40.22 & 82.57 \\
\hline 4 & qqlive & 93.88 & 35.88 & 52.93 & 88.81 \\
\hline 5 & PPstream & 90.53 & 41.73 & 44.58 & 86.31 \\
\hline 6 & Unknown P2P & 0 & 35.27 & 48.93 & 84.20 \\
\hline
\end{tabular}

but as collecting multi-hosts statistical characteristic needs a certain time, while statistical characteristic of the type of the other communicating party is real time, we can first detect the data packet whether it fit the Equation 2, then judge the Equation 1, as shown in the Figure 5.

In the laboratory, we run some common P2P software, part of which are based on known payload characteristics, and others whose load characteristics we do not know, such as BitTorrent, eMule, pplive, qqlive, PPstream, Kugoo on different hosts, and use normal WEB, FTP and other traditional network services on other 10 hosts, and run P2P-CNTIM prototype system and P2P identify payload-based system on the other 2 hosts. Set the detection time to 10 unit time, statistics ration of hosts identified P2P flow and total hosts generated P2P flow, repeat this experiment and calculate the mean value, then take it as the P2P identification accuracy rate. The experimental results are shown in Table 1.

The data from Table 1 can be seen, for the known type of P2P applications, the traditional payload-based identification system has higher recognition accuracy rate comparing with P2P-CNTIM prototype system, the main reason is that payload-based system detected all the pass data packet while P2P-CNTIM prototype system only can detect part of data packet because of the packets' available time and can be affected by complex network condition as well as user usage condition. All that can lead to a smaller P2P identification accuracy decreased. However, for unknown P2P stream, payloadbased detection methods cannot detect the P2P streaming, but P2P-CNTIM recognition system can still classify the $\mathrm{P} 2 \mathrm{P}$ streaming accurately, so to identify unknown P2P streaming, P2P-CNTIM identification system obviously has many advantages.

P2P-CNTIM model has a higher accuracy rate combining the multi-hosts characteristic with the type of the other communicating party characteristic comparing with using separate feature detection, as shown in Figure 9. This is mainly because for multi-hosts characteristic, if the application does not take up many P2P ports, or user 


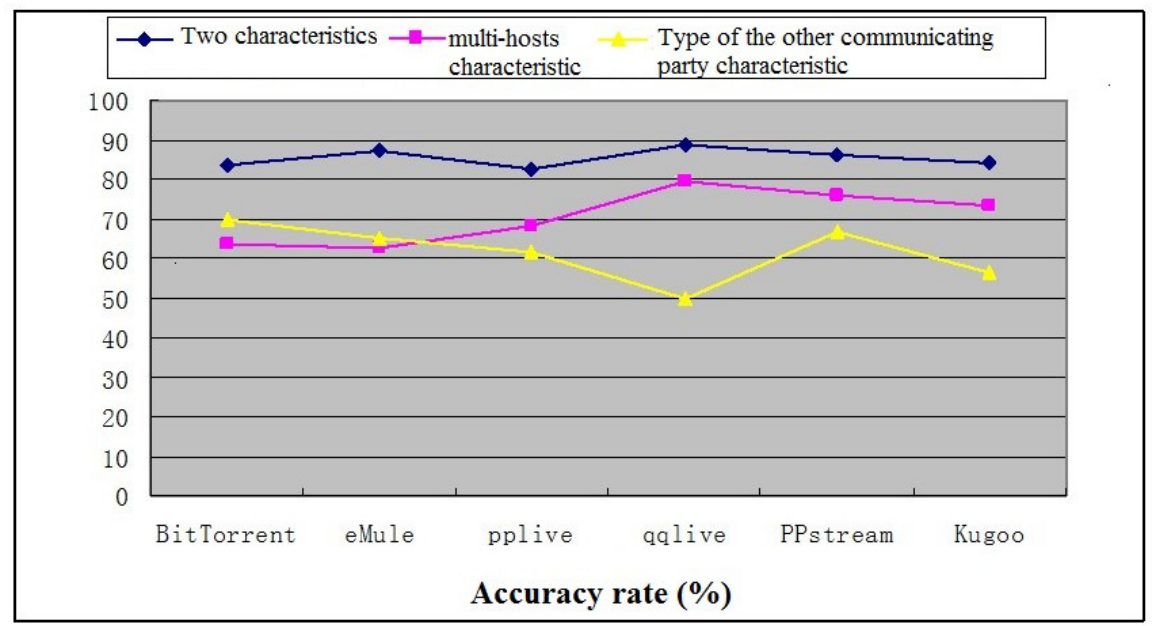

Figure 9. Accuracy rate contras.

limited the connection number, it is prone to miss some packets and for type of the other communicating party characteristic for P2P identification, perhaps some P2P applications use the low port to communicate, but as the rigor of algorithm, it also can result in missing. Therefore, the organic combination of two features for a common goal can get higher accuracy rate.

\section{Accuracy rate analysis and test}

P2P flow recognition systems generally deal with large amount of packet data, so efficiency will be the key indicator to measure a P2P identification system. To quantify the implementation efficiency of the comparison algorithms, we propose an abstract concept to measure the complexity of operation as basic operation unit which can form characteristic string matching. Assume in a period of time, the total number of treated unknown packets is $\mathrm{N}$, here the proportion of $\mathrm{P} 2 \mathrm{P}$ packet is $\mathrm{p}$, and the basic operation unit contained by all payload characteristic string in system is Kmax, then $\mathrm{N}$ packets implemented matching operation can be approximated with the formula below.

Opayload $=\mathrm{Ki}+\mathrm{Kmax}$

Where, Ki means basic operation unit number contained by match operation correspond to the i-th packet which matches a characteristic attribute.

Assume the P2P flow identified by the type of the other communicating party of P2P-CNTIM prototype system in the percentage of total P2P flows is $\mathrm{Pn}$, then for $\mathrm{N}$ packets operations performed can be approximately expressed as:

OP2P-CNTRM $=\mathrm{Li}+\mathrm{Mi}+\mathrm{Ki}+(\mathrm{Li}+\mathrm{Mi}) \mathrm{N}^{*}(\mathrm{Li}+\mathrm{Mi})+\mathrm{Ki}$

Where, Li means basic operation unit number contained by P2P identifying process based on type of the other communicating party characteristic to packet i, Mi means basic operation unit number contained by $\mathrm{P} 2 \mathrm{P}$ identifying process based on multi-hosts characteristic to packet $\mathrm{i}$. Because we only save the data packet in unit time, Li and Mi have upper limit.

In order to make a comparative study of P2P identification efficiency based on payload and type of the other communicating party characteristic, we assume that Equations 5, 6, then by equation 7 :

$\mathrm{N}^{*}(\mathrm{Li}+\mathrm{Mi})=\mathrm{N}^{*}(1-\mathrm{P})^{*} \mathrm{Kmax}$

$\mathrm{Li}+\mathrm{Mi}=(1-\mathrm{P})^{*} \mathrm{Kmax}$

$\mathrm{Kmax}=(\mathrm{Li}+\mathrm{Mi}) /(1-\mathrm{P})$

In Equation 7, when the proportion of P2P packet $\mathrm{p}$ is close to 1, then Kmax will be able to get great value, at this time, and lead that P2P identification based on payload has a higher efficiency than P2P-CNTIM recognition method. However, with the proportion of nonP2P packet mount up, the identification efficiency of P2P identification method based on P2P-CNTIM will be higher than the implementation P2P payload-based identification. Since Kmax is the basic operation unit contained by all payload characteristic string in system, it generally much larger than $\mathrm{Li}+\mathrm{Mi}$, and with the introduction of P2P flow control mechanism, P2P traffic on the network in proportion will gradually decrease, that can verify the implementation of the efficiency of P2PCNTIM recognition method will be superior to the traditional payload-based recognition method.

In this experiment, we ran P2P applications and non-P2P applications in different experimental hosts, and continuously improved the proportion of non-P2P flows, recorded results are shown in Figure 10.

\section{CONCLUSION}

The fast development of P2P technology had resulted in a number of problems. Along with the new P2P protocol 


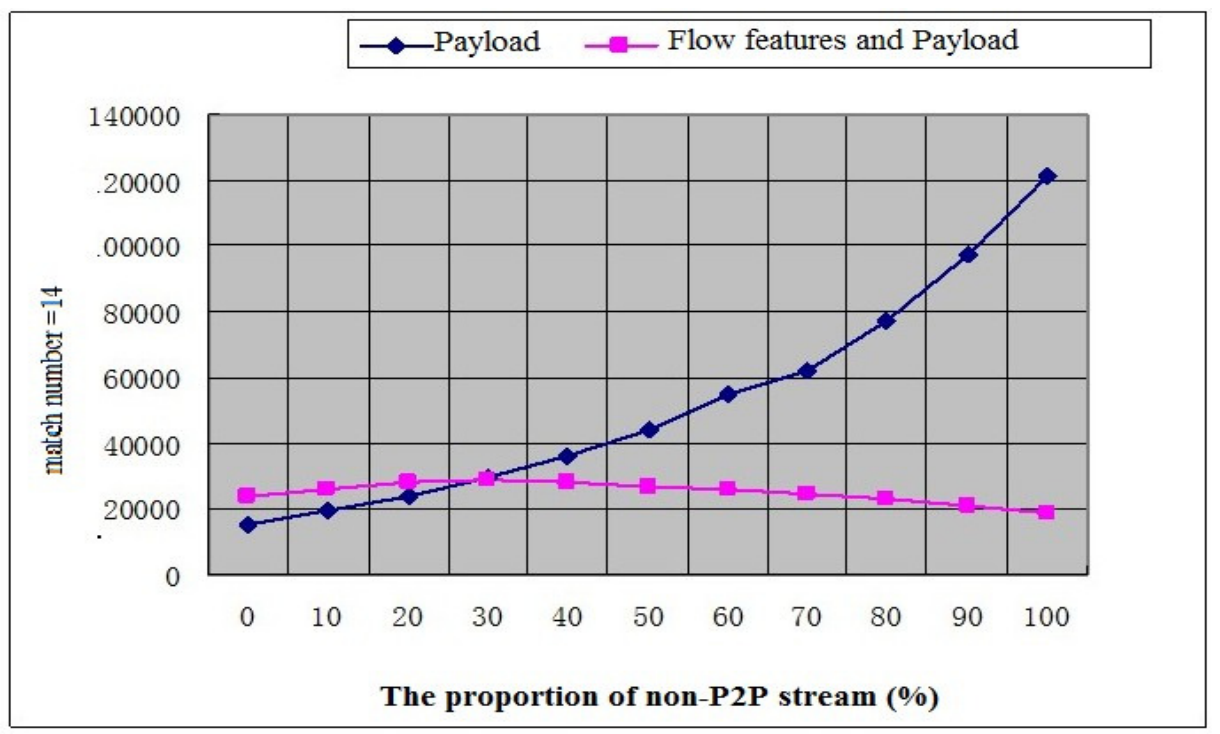

Figure 10. Comparison of the efficiency of P2P-CNTIM model and system based on Payload

and application emerging in multitude, the problem is getting worse. Thus how to classify the P2P stream efficiently and put them under control is becoming the urgent problem and we must solve them without hesitation. In this paper, according to the network topology, we establish an optimization model combining the multi-hosts characteristic with the type of the other communicating party characteristic in order to get a lower false-positive rate and higher accuracy and efficiency. We detailed the introduction of the model and its discriminant functions, scheduling mechanism and the core process. Finally, a simulation study testified the application and validity of the identification model and method. The simulation results are presented and analyzed in detail. Our future interests lie in developing a model based on host process while identifying P2P stream.

\section{ACKNOWLEDGMENTS}

This research work acknowledges the supports of the National Natural Science Foundation of China under Grant No.60973140, No.61003237, No.2009AA01Z212; the Natural Science Foundation of Jiangsu Province under Grant No. BK2009425; the Academic Natural Science Foundation of Jiangsu Province under Grant No. 08KJB520005, 10KJB510018; the Six Projects Sponsoring Talent Summits of Jiangshu Province and Innovation Project of JiangSu Province.

\section{REFERENCES}

Chunzhi Wang, Tao Li, Hongwei Chen (2009). P2P Traffic Identification Based on Double Layer Characteristics. In Proc. Information
Technology and Computer Science on IEEE Database. Comput. Sch., Hubei Univ. of Technol., Wuhan. China, pp. 593-596.

Feng Liu, Zhitang Li, Junfeng Yu (2009). P2P Applications Identification Based On the Statistics Analysis of Packet Length. In Information Engineering and Electronic Commerce on IEEE Database. Dept. of Comput. Sci. Technol., Huazhong Univ. of Sci. Technol. Wuhan, China.,pp. 160-163.

Feng Liu, Zhitang Li, Qingbin Nie (2009). A new method of P2P traffic identification based on Support Vector Machine at the host level. In Information Technology and Computer Science on International Conference. Dept. of Comput. Sci. \& Technol., Huazhong Univ. Sci. Technol., Wuhan, China .pp. 579-582.

Holger B, Erwin P, Rathgeb, Stefan Zilling (2006a). Advanced P2P Multi-protocol Traffic Analysis Based on Application Level Signature Detection. In Telecommunications Network Strategy and Planning Symposium on NETWORK. Inst. Exp. Math. , Duisburg-Essen Univ. Essen, pp. 1-6.

Holger B, Erwin P. Rathgeb, Stefan Zilling (2006b). Evaluation of an Efficient Measurement Concept for P2P Multi-protocol Traffic

Analysis. In Software Engineering and Advanced Applications. Inst. Exp. Math. , Duisburg-Essen Univ., Essen. pp. 414 - 423.

http://www.cnttr.com/114196/viewspace_5625.html

jing G, Zhixin S, Qiang G (2007). Research of Identification Method Based on P2P Flow Behavior Characterization. In Mini-Micro Systems on CNKI Database. College of Applied Mathematics \& Physics of Nanjing University of Posts and Telecommunications; Nanjing, College of Computer of Nanjing University of Posts and Telecommunications; Nanjing, China, pp. 48-53.

Peter Phaal. Detecting NAT Devices using sFlow[EB/OL]

Rui Yang (2007). The application of Characteristic string Match in P2P flow Control. In Science and Technology Information on CNKI Database. Mianyang Normal College, Sichuan, China, pp.158-159.

Subhabrata S, Jia W (2004). Analyze peer-to-peer traffic across large networks. On IEEE Database. AT\&T Labs.-Res., USA, pp. 219-232.

Thomas Karagiannis, Andre Broido, Michalis Faloutsos, KC Claffy (2004). Transport layer identification of $p 2 p$ traffic.

Xing Lu, Haixin Duan, Xing Li (2007). Identification of P2P Traffic Based on the Content Redistribution Characteristic. In Communications and Information Technologies on IEEE Database, Tsinghua Univ., Beijing, pp. $596-601$. 